\title{
Protestas sociales en Chile y tiempos líquidos. Una oportunidad para reformas urgentes en el campo de la salud pública
}

\section{Social protests in Chile and liquid times. An opportunity for urgent reforms in the field of public health}

\author{
Juan Pablo Murillo Peña ${ }^{1}$, Marcelo López Campillay ${ }^{2}$, Karen Pesse-Sorensen ${ }^{3}$ \\ ${ }^{1}$ Sección de Ciencias Sociales, Departamento de Medicina Preventiva y Salud Pública, Facultad de Medicina, Universidad Nacional Mayor de San Marcos. Lima, Perú. \\ ${ }^{2}$ Profesor Asistente, Doctor en Historia, Programa de Estudios Médicos Humanísticos, Facultad de Medicina, Pontifica Universidad Católica de Chile. Santiago de Chile, Chile. \\ ${ }^{3} \mathrm{MD}, \mathrm{MPH}, \mathrm{PhD}(\mathrm{c})$, Doctorado de Salud Pública, Facultad de Medicina, Universidad de Chile. Santiago de Chile, Chile.
}

\section{Correspondencia: \\ Juan Pablo Murillo Peña \\ jpmurillop@gmail.com}

Recibido: 3 de diciembre 2019

Aprobado: 16 de diciembre 2019

Publicaciónen línea: 28 de diciembre 2019

Conflictos de interés: Los autores declaran no tener conflictos de interés.

Citar como: Murillo JP, López M, Pesse-Sorensen K. Protestas sociales en Chile y tiempos líquidos. Una oportunidad para reformas urgentes en el campo de la salud pública. An Fac med. 2019;80(4):415-8. DOl: https:/doi. org/10.15381/anales.v80i4.17250
Este año 2019 el punto central de la agenda pública ha sido el creciente cuestionamiento de los sistemas democráticos. Diversas coyunturas políticas, sociales y económicas han desembocado en manifestaciones masivas. Paris, Hong Kong, Barcelona, recientemente Quito, La Paz y Santiago, han sido foco de grandes protestas ciudadanas. Como parte de este contexto, en el Perú, una grave crisis institucional, condujo a un cierre constitucional del Congreso de la República. Es evidente que como lo señalan varios estudios, podemos hablar de una crisis del sistema democrático a nivel global ${ }^{(1)}$.

Esta crisis global tiene muchos componentes. Uno de ellos, es la extraordinaria expansión de la desigualdad en el mundo, la cual incide directamente en los cuestionamientos a la democracia liberal a nivel global (2). Actualmente, ocho personas (ocho hombres en realidad) poseen la misma riqueza que 3600 millones de personas (la mitad de la humanidad). Los ingresos del 10\% más pobre de la población mundial han aumentado a menos de 3 dólares al año entre 1988 y 2011 , mientras que los ingresos del $1 \%$ más rico se han incrementado 182 veces más ${ }^{(3)}$. Desde diversas perspectivas, existe fuerte evidencia de que la estructura del capitalismo globalizado del siglo XXI genera una amplia desigualdad, lo cual se ha explicado con una fuerte base empírica y teórica desde diversas perspectivas ${ }^{(4,5,6)}$. La capacidad de erosión de la trama social, generada por la desigualdad, unida a la valoración de la competencia y por ende, el individualismo, en esta etapa del capitalismo global y su capacidad de generar amplias capas de exclusión, incluso en los países del primer mundo, ha sido extensamente documentada ${ }^{(7,8,9)}$.
Sin embargo, las protestas en Chile en términos de su magnitud y radicalidad han llamado particularmente la atención en toda América Latina, dado el crecimiento de la economía chilena en las últimas décadas, su amplia apertura a los mercados y su ingreso a la Organización para la Cooperación y el Desarrollo Económicos (OCDE); hitos que convirtieron a este país en un referente en la implementación de las reformas de libre mercado en América Latina.

Así, en el escenario académico chileno, desde diversas perspectivas, se ha remarcando la necesidad de importantes reformas en diversos ámbitos de la sociedad chilena ${ }^{(10,11)}$, además de identificar importantes problemas de la democracia chilena, como los denominados "Enclaves de la transición" (12). Uno de estos espacios académicos, fue el coloquio organizado por la Facultad de Medicina de la Universidad Católica de Chile: "Salud Pública en el siglo XXI: ¿Nuevas interrogantes en tiempos líquidos? Experiencias iberoamericanas". En este evento se discutió la necesidad de incorporar a la salud pública enfoques teóricos alternativos, en un contexto de crisis de la globalización neoliberal. En ese evento se analizó, desde diversas miradas, cómo el pensamiento de Zygmunt Bauman a partir de su concepto de "modernidad líquida" nos permitía entender las diversas tensiones que se apreciaban en nuestras sociedades ${ }^{(13)}$. Se discutió ampliamente la necesidad de reconstruir el tejido social a partir recuperar el sentido republicano y solidario de nuestras democracias, entendidas como un proceso de negociación y reconciliación, que expresa una noción de vida compartida ${ }^{(14)}$. Un elemento que se planteó en las discusiones del evento, fue la extremada rigidez de las 
estructuras sociales y estatales chilenas. Si bien eran claras y explícitas las tensiones expresadas en un conjunto de problemas urgentes de abordar desde el campo de la salud pública, como la creciente desigualdad y exclusión de algunos segmentos de la sociedad, no se atisbaba en ese momento el carácter disruptivo del actual escenario de protestas.

¿Qué es lo que se rompió finalmente en la sociedad chilena para liberar esa explosión de movilización ciudadana?. Consideramos que un elemento central es la fractura del esquema de gubernamentalidad neoliberal, la crisis de red de protección social chilena. La sensación de desprotección colectiva, frente a los importantes niveles de segmentación y exclusión con enormes brechas de inequidad (la diferencia de esperanza de vida entre una mujer que vive en un área urbana pobre de Santiago respecto a una que vive en un área rica es de 17,7 años) ${ }^{(15)}$, sumados a la privatización de los riesgos, los cuidados y los bienes públicos (incluso el agua), generaron un sentimiento de precarización de la vida intolerable para una porción significativa de la sociedad chilena ${ }^{(16)}$. Todo ello se tradujo en la demanda social de una vida digna, del derecho a ser visto y tratado como a una persona, como un igual, como un ciudadano de una república, tal como lo expresa la siguiente frase que señala el objetivo de las movilizaciones: "Hasta que la dignidad sea costumbre".

Un elemento que ha llamado poderosamente la atención es la centralidad de las críticas al sistema de salud chileno en las protestas ${ }^{(17)}$, en las cuales aparecen problemas señalados hace mucho tiempo: atención médica estratificada socialmente, dificultades de financiación y gestión de recursos, falta de acceso a los servicios y al tratamiento, fragmentación de servicios de salud, normas e instituciones, la medicalización del concepto de salud (resolver enfermedades más que acentuar prevención), sistema universitario orientado a la formación de profesionales especialistas y no en agentes de salud, etc. El escenario de las protestas, ha permitido visibilizar la existencia de diversas propuestas de reforma del sistema de salud, las cuales no habían encontrado una voluntad política para ponerlas en discusión ${ }^{(18,19)}$.
El actual momento constituyente en Chile, que fue posible por las masivas manifestaciones populares, implica el retorno a un debate que comenzó en la década de 1920 y colocó a la protección en salud en un lugar importante en el proyecto de república ${ }^{(20,21)}$. Este debate se hizo más intenso en las décadas de 1960 y 1970 donde se esbozó una aspiración de construir un sistema efectivamente universal en salud. Si bien en el actual escenario hay varias propuestas de reforma al sistema de salud chileno que responden a un escenario de discusión previa al escenario de protestas, donde ha ganando consenso la idea de un sistema universal y equitativo; sin embargo, la discusión de un nuevo pacto constitucional, implica una discusión de mayor densidad: la oportunidad de establecer el logro del derecho a la salud como un deber del estado, a partir de un principio de solidaridad y la construcción de un nuevo estado social, tal como la entendemos en el contexto del siglo XXI ${ }^{(22,23)}$. Ello plantea un marco amplio de discusión en torno a sus implicancias en lo que entendemos por salud, que excede ampliamente del ámbito biomédico y que plantea definir el conjunto de bienes públicos que son necesarios para garantizar esta aspiración, ello en un contexto donde el debate en torno a la contaminación ambiental, el cambio climático, la seguridad alimentaria y la sostenibilidad de los sistemas hídricos andinos adquieren una enorme importancia, lo que conlleva a una nueva noción de la salud como horizonte de acción, decididamente transversal a todas las políticas públicas. Esto también implica pensar en una institucionalidad estatal y en una tecnología de gobierno radicalmente distinta a la existente.

Este proceso será todo un desafío, la gran emergencia a lo largo de Chile, de cabildos ciudadanos de discusión sobre la nueva constitución y las esperadas reformas del sistema de salud, plantean la necesidad de una gran capacidad de diálogo sin exclusiones, que recoja las propuestas del conjunto de la ciudadanía y sea capaz de integrar dichas demandas con las agendas de las corporaciones profesionales, que en estas coyunturas suelen hegemonizar el debate, orientándolo a una visión biomédica y por ende reduccionista de la salud y el bienestar. Tomará tiempo implementar los arreglos sistémicos que deriven de este proceso constituyente, y ello implicará una enorme tensión entre las expectativas de los diversos actores sociales y la realidad de los servicios de salud local, donde los estudios muestran la desesperanza y escepticismo de ciudadanos y profesionales, respecto a la posibilidad de lograr cambios reales en las formas de funcionamiento de los servicios locales de salud.

En entrevistas realizadas a profesionales y pacientes, en el contexto del trabajo actualmente en curso, realizado por uno de los autores en Chile sobre calidad de atención, se recogieron múltiples expresiones de este descontento:

Por ejemplo, una asistente social dice: "nos encontramos con un caso, una señora de casi 90 años, que vive... vive sola en el cerro, en condiciones ultra, ultra infrahumanas, una viejita que... las cosas bien de salud no me la sé, pero el doctor la ha ido a ver, y yo creo que tiene presión alta, debe tener todas esas cosas. Pero nosotros... yo he pasado como dos años y que no hemos podido mejorarle su calidad de vida, su entorno".

Y un médico reclama un derecho que debería ser obvio: "Si no se legisla en razón a la obligatoriedad que debería tener un empleador en darle el tiempo como para poder asistir a un control de salud, sin que se vea amedrentado, sancionado. ¿Ya? Pucha, sería, espectacular".

Un paciente siente que: "Estamos en un minuto, en un sistema donde la humanidad no existe, somos simplemente números. Y para quién sea, ah. Yo critico este país, critico a la política de este país, independiente sea de derecha izquierda o centro, no me importa. ¿Por qué? Porque todos quieren plata, no quieren otra cosa, no miran hacia el pueblo". Otra paciente recuerda una larga espera en la urgencia de un hospital público buscando atención para su abuela mayor de 85 años que la llevó a recurrir al sistema privado: "Mi mami estaba aquí, mi hermana estaba allá y mi mami aquí tenía la cabeza de ella, así. No, no quería ni moverse y se quejaba y se quejaba. Así que llegó justo mi tío y a él le dije: No tío, vámonos pa' la clínica. Si ahí nos arreglamos como pagamos no más." 
En el Perú, el escenario constituyente en Chile ha despertado una gran expectativa, dada la existencia de un escenario de economía de mercado con patrones de marcada desigualdad, donde el $1 \%$ de la población de mayores ingresos concentra el $38,7 \%$ de los ingresos ${ }^{(24)}$, un perfil de reformas similares en el campo económico y la liberalización de aspectos importantes de los mecanismos de protección social como la educación y pensiones. Además, el Perú comparte con Chile un proceso de implementación sumamente riguroso de las políticas emanadas del "Consenso de Washington", las cuales en ambos países se realizaron en un contexto político autoritario (25). Ello ha despertado un gran interés en el debate chileno en torno a la nueva constitución en general y en particular sobre las demandas de reformas en el sector salud, las cuales podrían constituir una ventana de oportunidad, para impulsar cambios importantes en las políticas públicas de salud en el Perú.

Sin embargo, a pesar de las semejanzas respecto al proceso chileno, la sociedad peruana está lejos todavía de impulsar cambios cualitativos importantes en las políticas de salud. En Chile el actual debate sobre el futuro proceso constitucional muestra una acumulación de masa crítica importante para sostener procesos de cambio, aun con un enorme desprestigio de clase política, tanto a nivel de capital social, intelectual, institucional, incluso simbólico, que además responden a una fuerte tradición republicana que ha caracterizado a la sociedad chilena en el siglo XX. En el Perú, lo que ha predominado, en un escenario posterior al colapso de los partidos políticos, es la precarización de los liderazgos personalistas y clientelares, la construcción de identidades políticas a partir de la desafección y una enorme dificultad para agregar intereses ${ }^{(26)}$. Este proceso se ha acompañado en la última década, de un creciente proceso de deterioro de las capacidades institucionales del Estado ${ }^{(27)}$ el cual en algunos ámbitos está desbordado por la sociedad y en donde, en algunos casos se han desdibujado las diferencias entre el espacio público y el espacio privado ${ }^{(28)}$ planteándose incluso una "captura del Estado" por parte de in- tereses privados ${ }^{(29)}$. Asimismo, se ha documentado una enorme debilidad de las tecno-burocracias para impulsar y sostener procesos de reforma en salud (30). Asimismo, desde hace varias décadas se ha planteado un escenario de crisis en la matriz de pensamiento sanitario peruano (31), el cual todavía representa un tema sin resolver y constituye un obstáculo en un escenario complejo como el actual que reclama una intensa renovación conceptual de cara a los retos que enfrenta el sistema de salud peruano en el siglo XXI.

El complejo escenario social y político del Perú, plantea un conjunto de dificultades estructurales para seguir avanzando en el proceso de reformas de salud, que tienen que abordarse con mucho rigor y honestidad intelectual, haciendo un balance de todos los avances y retrocesos en el sector salud en la última década; construir una visión compartida de la magnitud y complejidad de los desafíos e identificar consensos respecto a las intervenciones de reforma que el sector salud peruano está en condiciones de sostener. Pero sobre todo, pasar a discutir muy sinceramente el conjunto de capacidades y acumulaciones que se requieren para poder diseñar e impulsar cambios significativos, viables y sostenibles en el sector salud peruano.

Todo ello nos obliga a moderar expectativas en ambos lados de la frontera: en Chile, desde la efervescencia social, viene un proceso largo y complejo, con discusiones muy trascendentes, pero que van a tomar tiempo para su traducción en políticas públicas que incidan en la vida cotidiana de los ciudadanos. Por el lado peruano, desde la precariedad institucional, se requiere construir una visión compartida en el sector del tipo de reformas que son posibles de sostener en este periodo, mientras se configura el escenario de discusión política para las elecciones del 2021, donde se debería tener clara una visión de los problemas estructurales del sistema de salud peruano y del conjunto de políticas públicas posibles para su transformación en la siguiente década. En ese sentido, en ambos lados de la frontera se requerirá construir a partir de "liderazgos heroicos", parafraseando a Miztberg ${ }^{(32)}$, de personas o grupos sociales de diferentes ámbitos, fuertemente comprometidos con la construcción de los difíciles consensos y acuerdos políticos necesarios para alcanzar los objetivos que demandan la coyuntura histórica particular de cada país.

\section{REFERENCIAS BIBLIOGRÁFICAS}

1. Mounk Y, Foa RS. The End of the Democratic Century. Foreign Affairs. 2018; 97(3):29-38.

2. Mounk Y. El pueblo contra la democracia. Por que nuestra libertad está en peligro y como salvarla, 1ra. Edición. Barcelona: Editorial Paidos; 2018.

3. Oxfam [Internet]. Una Economía para el $99 \%$. Es hora construir una economía más humana y justa al servicio de las personas, 2017 [Fecha de acceso: 3 de diciembre 2019]. Disponible en: https://www. oxfam.org/es/informes/una-economia-para-el-99

4. Piketty T. El Capital en el siglo XXI 1 ra. Edición. Santiago de Chile: Fondo de Cultura Económica; 2014.

5. Bogosian B. The Inescapable Casino. Scientific American. 2019;321(5):70-77. DOI: 10.1038/ scientificamerican1119-70

6. Stiglitz J. La gran brecha, 1ra. Edición. México: Penguin Random House Grupo Editorial; 2017.

7. Durand PV. Desigualdad Social y Ciudadania Precaria. ¿Estado de excepción permanente?. México: Instituto de Investigaciones Sociales/Siglo XXI Editores; 2010.

8. Sassen S. Expulsiones. Brutalidad y complejidad de la Economia Global, 1ra Edición. Madrid: Katz Editores; 2015

9. Bauman Z. Daños Colaterales. Desigualdades sociales en la era global, 1ra Edición. México: Fondo de Cultura Económica. 2011:59-74.

10. Atria F, Larrain G, Benavente J, Couso J, Joignant A. El otro modelo. Santiago de Chile: Ramdom House Mondadori; 2013

11. Mayol A. El derrumbe del modelo. La crisis de la economía de mercado en el Chile contemporáneo. Santiago de Chile: Editorial LOM; 2012.

12. Yasui S. Transición y consolidación democrática en Chile: un balance crítico de 20 años. En Murakami Y. (Editor). América Latina en la era posneoliberal: democracia, conflictos y desigualdad. CIAS-IEP, Lima 1ra Edición 2013, páginas 87-109.

13. Facultad de Medicina, Universidad Católica de Chile. Coloquio: "Salud Pública en el siglo XXI: ¿Nuevas interrogantes en tiempos líquidos?. Experiencias iberoamericanas". Santiago de Chile, 26 de Setiembre del 2019.

14. Bauman Z. Modernidad Liquida. México D.F.: Fondo de Cultura Económica. 2003: p. 189.

15. Bilal U, Alazraqui M, Caiaffa W, Lopez-Olmedo N, Martinez-Folgar K, Miranda J. Inequalities in life expentancy in six large Latin American cities from SALURBAL study: an ecological análisis. The Lancet Planetary Health. 2019;3(12):E503-E510. DOI: https://doi.org/10.1016/S2542-5196(19)30235-9

16. Lorey I. Estado de inseguridad. Gobernar la precariedad. Madrid: Ed. Traficantes de sueños; 2016.

17. Fraser B. Violent protest in Chile linked to health-care inequities. The Lancet. 2019;394(10210):16971698. DOI: 10.1016/S0140-6736(19)32720-5

18. Propuesta de modernización y fortalecimiento de 
prestadores estatales de los servicios de salud. Centro de Estudios Públicos-Escuela de Salud Pública Salvador Allende, Universidad de Chile 2017.

19. Escuela de Salud Pública de la Universidad de Chile, Colegio Médico de Chile. Propuestas para una reforma integral al financiamiento de la salud en Chile. Santiago de Chile: Escuela de Salud Pública Salvador Allende, Universidad de Chile; 2018.

20. Allende S. La realidad médico-social chilena. Santiago de Chile: Universidad de Chile; 1939 DOI: https://doi.org/10.34720/mj3z-6m24

21. Camus $P$, Zúñiga HF. La Salud Pública en la Historia de Chile. Anales Chilenos de Historia de la Medicina. 2017;17(2):155-175.

22. Ballesteros C. Economía a escala humana. Una aproximación a los valores de la economía solidaria desde las ideas de Max-Neff. Nuevas Tendencias en Antropología. 2010;(1):89-107.

23. Slachevsky AN, Cubillos CP, Yañez RR. El Estado social del mañana: diálogos sobre el bienestar democracia y capitalismo con Göran Therborn,
Phillipe Van Parijs y Nicolas Duvoux, 1a Edición. Santiago de Chile: LOM Ediciones; 2019.

24. Cruz M, Seminario B, Campos C. Desigualdad (Re)considerada Peru 1997-2015. Journal of Economics, Finance and International Business. 2018;2(1):12-52. DOI: http://dx.doi.org/10.20511/ jefib.2018.v2n1.219

25. Morandé F. A casi cuatro décadas del Consenso de Washington ¿Cuál es su legado en América Latina?. Estudios Internacionales. 2016;185:31-58.

26. Meléndez C. El mal menor. Vínculos políticos en el Perú posterior al colapso del sistema de partidos, 1ra. Edición. Lima: Instituto de Estudios Peruanos; 2019

27. Vergara A. ¿Hortelanos o Republicanos? En Ciudadanos sin República. De la precariedad institucional al descalabro político. Prólogo a la segunda edición. Lima: Ed. Planeta. 2018: 13-30.

28. Grompone R. El Estado como institución afirmada y desbordada por la sociedad. En Incertidumbres y distancias. El controvertido protagonismo del Estado en el Perú. Romeo Grompone (Editor), 1ra. Edición. Lima: Instituto de Estudios Peruanos. 2016: 27-93.

29. Durand F. La Mano Invisible del Estado. Efectos del neo liberalismo en el empresariado y la política, 1ra. Edición. Lima: Fundación Friedrich Ebert; 2005.

30. Chaname C. Implementing Public Policy in "Democracies without Parties". Why does policy implementation in developing countries fail? The case of Healthcare Policy Reform in Peru: 2012-2015 (Tesis para el grado de MPA FT Public Administration, Queen Mary School of Business and Management, University of London); 2019.

31. Murillo J, Mendoza W, Franco G. ¿Quo vadis Salud Pública. Discurso y práctica de la Salud Pública en el Perú y su auge universitario en la década de los noventa. An Fac med. 2000;61(2):146-160. DOI: https://doi.org/10.15381/anales.v61i2.4341

32. Mintzberg H. Managing the myths of health care. Bridging the separations between care, cure, control and community, First Edition. Oakland CA: Berrett-Koehler Publishers; 2017. 